\title{
The prevalence of women's emotional and physical health problems following a postpartum haemorrhage: a systematic review
}

Margaret Carroll ${ }^{1 *}$ D, Deirdre Daly ${ }^{1}$ and Cecily M. Begley ${ }^{1,2}$

\begin{abstract}
Background: Postpartum Haemorrhage (PPH) is a leading cause of maternal mortality with approximately 225 women dying as a result of it each day especially in low income countries. However, much less is known about morbidity after a PPH. This systematic review aimed to determine the overall prevalence of emotional and physical health problems experienced by women following a postpartum haemorrhage.
\end{abstract}

Methods: Eight databases were searched for published non-randomised, observational, including cohort, primary research studies that reported on the prevalence of emotional and/or physical health problems following a PPH. Intervention studies were included and data, if available, were abstracted on the control group. All authors independently screened the papers for inclusion. Of the 2210 papers retrieved, six met the inclusion criteria. Data were extracted independently by two authors. The methodological quality of the included studies was assessed using a modified Newcastle Ottawa Scale (NOS). The primary outcome measure reported was emotional and physical health problems up to 12 months postpartum following a postpartum haemorrhage.

Results: Two thousand two hundred ten citations were identified and screened with 2089 excluded by title and abstract. Following full-text review of 121 papers, 115 were excluded. The remaining 6 studies were included. All included studies were judged as having strong or moderate methodological quality. Five studies had the sequelae of PPH as their primary focus, and one study focused on morbidity postnatally, from which we could extract data on PPH. Persistent morbidities following PPH (at $\geq 3$ and $<6$ months postpartum) included postnatal depression (13\%), post-traumatic stress disorder (3\%), and health status 'much worse than one year ago' (6\%). Due to the different types of health outcomes reported in the individual studies, it was possible to pool results from only four studies, and only then by accepting the slightly differing definitions of PPH. Those that could be pooled reported rates of acute renal failure (0.33\%), coagulopathy $(1.74 \%)$ and re-admission to hospital following a PPH between 1 and 3 months postpartum (3.6\%), an appreciable indication of underlying physical problems.

Conclusion: This systematic review demonstrates that the existence and type of physical and emotional health problems post PPH, regardless of the volume of blood lost, are largely unknown. Further large cohort or case control studies are necessary to obtain better knowledge of the sequelae of this debilitating morbidity.

Keywords: Postpartum haemorrhage, Systematic review, Emotional health problems, Physical health problems

Abbreviations: CS, Caesarean section; DIC, Disseminated Intravascular Coagulation; NOS, Newcastle Ottawa Scale; $\mathrm{PPH}$, Postpartum haemorrhage

\footnotetext{
* Correspondence: carrolol@tcd.ie

'School of Nursing and Midwifery, Trinity College Dublin, 24 D'Olier Street,

Dublin DO2 T283, Ireland

Full list of author information is available at the end of the article
} 


\section{Background}

Postpartum Haemorrhage $(\mathrm{PPH})$ is recognised as one of the leading causes of maternal mortality worldwide, accounting for 1 in 4 maternal deaths globally, but much less is known about morbidity post PPH. At least 225 women die from PPH every day, the majority of these deaths occurring in low income countries [1]. In the most recent Confidential Enquiries into Maternal Deaths (2011-13) in the UK, PPH was identified as being responsible for $10 \%$ of direct maternal deaths [2]. Historically, maternal mortality has been used as an indicator of the quality of maternity services; however, as women's general health improves, and preventative care and treatment for PPH become more accessible, more women are surviving $\mathrm{PPH}$, making the evaluation of morbidity a useful complementary measure.

Although PPH is itself a maternal morbidity, the outcomes for women who experience a PPH have not been studied widely. A surveillance study conducted in 27 obstetric units in Brazil found the maternal near miss ratio to be 2.2 per 1000 women with PPH [3]. Wagner et el. [4] describe a review of 11 studies, showing that PPH resulted in anaemia with a summary ratio of 2.35 (95\% CI 1.44-3.84), albeit with considerable heterogeneity. Emotional outcomes occur also, with the majority of women who experience $\mathrm{PPH}$ having negative memories of the birth including, for some, a persistent fear of dying [5]. A systematic review of women's experiences of all types of severe maternal morbidity showed that women found these incidents physically and emotionally distressing [6], and therefore worthy of study.

The prevalence of $\mathrm{PPH}$ varies, with the worldwide prevalence thought to be between 6 and $11 \%$ [7]. In England, the incidence increased from $7 \%$ in 2004/5 to $13 \%$ in 2011/12 [8]. In an eleven-year population-based cohort study in Ireland, the rate of $\mathrm{PPH}$ increased from $1.5 \%$ in 1999 to $4.1 \%$ in 2009 [9]. In Australia (NSW), the $\mathrm{PPH}$ rate increased from $6.1 \%$ in 2003 to $8.3 \%$ in 2011 [10].

More recently, the rate in one large Dublin maternity hospital was $14.6 \%$ in 2014, a 4-fold increase from the $3.3 \%$ incidence reported in 2008 [11]. Whilst some of these increases may be a result of more accurate estimation of blood loss, or because $\mathrm{PPH}$ was redefined with a lower threshold of blood loss, it never-the-less means that more women are experiencing $\mathrm{PPH}$.

Given the prevalence of $\mathrm{PPH}$ and approximately 135 million births occurring worldwide each year, approximately 8-11 million women will experience a PPH and many will suffer some health sequelae. The focus of this systematic review, therefore, was on determining the overall prevalence of emotional and physical health problems experienced by women following a postpartum haemorrhage.

\section{Defining postpartum haemorrhage}

$\mathrm{PPH}$ is defined in four different ways by WHO [1], RCOG [12], ICD-10 codes [13] and NICE [14]. Primary (sometimes referred to as immediate or early) occurs within $24 \mathrm{~h}$ of birth, whilst secondary (sometimes referred to as delayed or late) occurs after $24 \mathrm{~h}$ and up to 12 weeks postpartum.

In these four definitions, the common component is the inclusion of blood loss greater than $500 \mathrm{mls}$ for a primary PPH. For secondary PPH, only the ICD-10 codes [13] categorise blood loss as being greater than $500 \mathrm{mls}$. The terms used in two other definitions state 'abnormal or excessive bleeding from the birth canal' [12] / or 'of the amount that adversely affects the maternal physiology' [13]. WHO [1] or NICE [14] do not define secondary PPH.

Other terms used to define $\mathrm{PPH}$ include major obstetric haemorrhage, defined not only according to blood loss (>2500 mls), but also by number of transfused units of blood $(n=5)$ or requiring coagulopathy treatment (e.g., fresh frozen plasma, fibrinogen concentrate substitution therapy, platelets) $[15,16]$.

However, despite the definition used, being able to estimate blood loss accurately is recognised as being almost clinically impossible [17], and studies have shown that blood loss is overestimated at low volumes and underestimated when the volume is large thus underestimating the severity of the PPH [18-20].

\section{Defining maternal morbidity - women's physical and emotional health problems}

The term 'maternal morbidity' encompasses a wide range of acute and chronic conditions, ranging from 'near death' or 'near miss' to non-life threatening events. A WHO review, undertaken to create a consensus around a definition of maternal morbidity, found significant discrepancies in the literature and amongst experts [21]. More recently, population based studies have reported prevalence of severe maternal morbidity and, according to these reports, 1 in 140 pregnant women will suffer from a severe morbidity, the most common being $\mathrm{PPH}$ [16]. In Ireland, major obstetric haemorrhage was also the most common cause of severe maternal morbidity, with a rate of 2.55 per 1000 maternities [15]. These rates are for severe haemorrhage only, meaning that the prevalence of any degree of $\mathrm{PPH}$ in the wider maternity population is much greater.

\section{Methods}

\section{Aim and objectives}

This systematic review aimed to determine the overall prevalence of emotional and physical health problems experienced by women following a postpartum haemorrhage. The objectives were:

To determine the prevalence of women's emotional health problems following a postpartum haemorrhage 
up to 3 months, $>3$ months and $\leq 6$ months, and $>6$ months and $\leq 12$ months postpartum; and

To determine the prevalence of women's physical health problems following a postpartum haemorrhage up to 3 months, $>3$ months and $\leq 6$ months, and $>6$ months and $\leq 12$ months postpartum.

\section{Outcomes}

Primary outcome measures were the reported emotional and physical health problems up to 12 months postpartum following a postpartum haemorrhage. The type of haemorrhage experienced included: 1 . Primary / immediate PPH; 2. Secondary / delayed PPH; and 3. PPH according to definition (as defined by WHO [1], RCOG [12], ICD-10 codes [13] and NICE [14]). When studies did not cite any of the above references, their definition was abstracted and assessed.

Secondary outcome measures were women's physical and/or emotional health problems following PPH according to parity, age, BMI and mode of birth and amount of blood lost/severity of PPH.

\section{Inclusion and exclusion criteria}

Inclusion and exclusion criteria were determined a priori. Eligible participants were postpartum women, irrespective of parity, who had given birth to a baby of at least 24 weeks gestation and who experienced a postpartum haemorrhage. An individual study had to report primary data on prevalence of emotional and/or physical health problems at any time point up to 12 months postpartum, to be included.

Published non-randomised, observational including cohort primary research studies, reporting on prevalence of emotional and/or physical health problems following a PPH were included. Intervention studies were included and data, if available, were abstracted on the control group.

Case reports, reviews and qualitative studies were excluded.

\section{Search and selection strategy}

A computerised search of the following electronic databases was performed from their foundation to April 2015: CINAHL , PubMED (MEDLINE not searched as included in PubMED), Maternity and Infant Care, PsycINFO, EMBASE (also includes deduplicated MEDLINE citations from 1966 to the present), Web of Science, Social Science Index and The Cochrane Library. Key words, search terms and string were developed, tested and adapted for each database. The search was not restricted by year or English language publications. Search terms were developed for the three key terms: 'postpartum haemorrhage', 'postnatal period' and 'women's emotional and physical health' (Table 1). Search strings for individual databases were created (Additional file 1: Search Strings for Databases).

Following an initial scoping search, the search was limited to 'title' OR 'abstract'. The appropriate search terms were combined with the Boolean operands 'AND' and 'OR' as appropriate.

Reference lists of papers retrieved for full text review were also reviewed for potentially eligible papers that may not have been captured by the electronic search strategy [22].

\section{Data management processes}

The review followed the processes recommended by Higgins and Green [23]. Following agreement on the final search string, the selection of eligible studies and data extraction were performed by three people independently (MC, $\mathrm{CB}$ and $\mathrm{DD})$, and the final results compared, thus minimising the likelihood that errors would go undetected. MC, CB and DD have expertise in midwifery, $\mathrm{CB}$ has systematic review and statistical expertise, DD has systematic review expertise.

\section{Quality assessment of included studies}

The Newcastle Ottawa Scale (NOS) [24] was modified and a scale of 0-6 stars was used to assess methodological quality of included studies (Table 2). Six stars was deemed as strong, four to five stars was deemed as moderate and three or fewer stars was deemed as weak methodological quality. Studies judged to have weak methodological quality were excluded from the data extraction process. All items were regarded as equally important, and no weighting was applied to any item or category.

\section{Data extraction management}

The following data were abstracted and inserted in a data collection form, designed a priori.

\section{Primary outcome measures}

i. Country of study, study design, date of data collection, and methods used

ii. Definition of PPH, if any, used.

The number of women reported as having a PPH, and who also reported a particular physical and/or emotional health problem, and the postpartum time it was reported.

\section{Data analysis}

Where possible, data reported on similar outcomes at the same time points were pooled. 
Table 1 Key words and search terms and string

\begin{tabular}{ll}
\hline Key words & Search terms/String \\
\hline Postnatal period & TI postnatal OR AB postnatal OR TI post natal OR AB post natal OR TI post-natal OR AB post-natal OR TI postpartum \\
& OR AB postpartum OR TI post partum OR AB post partum OR TI post-partum OR AB post-partum OR TI "postnatal \\
& period" OR AB "postnatal period" OR TI "post natal period" OR AB "post natal period" OR TI "post-natal period" OR \\
AB "post-natal period" OR TI "postpartum period" OR AB "postpartum period" OR TI "post partum period" OR AB & "post partum period" OR TI "post-partum period" OR AB "post-partum period" OR TI puerperium OR AB Puerperium \\
& OR TI peripartum OR AB peripartum OR TI peri partum OR AB peri partum OR TI peri-partum OR AB peri-partum OR \\
& TI "peripartum period" OR AB "peripartum period" OR TI "peri partum period" OR AB "peri partum period" OR TI \\
"peri-partum period" OR AB "peri-partum period" OR TI peripartum period OR AB peripartum period
\end{tabular}

Postpartum haemorrhage $\quad$ TI PPH OR AB PPH OR Tl postpartum haemorrhage OR AB postpartum haemorrhage OR TI post partum haemorrhage OR AB post partum haemorrhage OR TI post-partum haemorrhage OR AB post-partum haemorrhage OR TI postpartum hemorrhage $O R A B$ postpartum hemorrhage OR TI post partum hemorrhage OR AB post partum hemorrhage OR TI post-partum hemorrhage OR AB post-partum hemorrhage OR TI obstetric haemorrhage OR AB obstetric haemorrhage OR TI obstetric hemorrhage OR AB obstetric hemorrhage OR TI major obstetric haemorrhage OR AB major obstetric haemorrhage OR TI major obstetric hemorrhage OR AB major obstetric hemorrhage OR TI massive haemorrhage OR AB massive haemorrhage OR TI massive hemorrhage OR AB massive hemorrhage OR TI massive obstetric haemorrhage OR $A B$ massive obstetric haemorrhage OR TI massive obstetric hemorrhage OR AB massive obstetric hemorrhage OR TI severe haemorrhage OR AB severe haemorrhage OR TI severe hemorrhage OR AB severe hemorrhage OR TI severe obstetric haemorrhage OR AB severe obstetric haemorrhage OR TI severe obstetric hemorrhage OR AB severe obstetric hemorrhage OR TI severe blood loss OR AB severe blood loss

Women's emotional and physical TI women's health OR AB women's health OR TI womens health OR AB womens health OR TI woman's health health problems

\section{OR AB woman's health OR TI womans health OR AB womans health OR TI health outcome OR AB health outcome} OR TI health outcomes OR AB health outcomes OR TI adverse outcome OR AB adverse outcome OR TI adverse outcomes $O R A B$ adverse outcomes OR TI pregnancy outcome OR AB pregnancy outcome OR TI pregnancy outcomes OR AB pregnancy outcomes OR TI puerperal disorder OR AB puerperal disorder OR TI puerperal disorders OR AB puerperal disorders OR TI maternal morbidity OR AB maternal morbidity OR TI maternal morbidities $O R A B$ maternal morbidities OR

TI mental health OR AB mental health OR TI mental disorder OR AB mental disorder OR TI mental disorders OR AB mental disorders OR TI mental disease OR AB mental disease OR TI mental diseases OR AB mental diseases OR TI emotional wellbeing OR AB emotional wellbeing OR TI emotional well being OR AB emotional well being OR TI emotional well-being OR AB emotional well-being OR TI emotional health OR AB emotional health OR TI postnatal depression OR AB postnatal depression OR TI post natal depression OR AB post natal depression OR TI post-natal depression OR AB post-natal depression OR TI postpartum depression OR AB postpartum depression OR TI post partum depression OR AB post partum depression OR TI post-partum depression OR AB post-partum depression OR TI postnatal distress OR AB postnatal distress OR TI post natal distress OR AB post natal distress OR TI post-natal distress OR AB post-natal distress OR TI postpartum distress OR AB postpartum distress OR TI post partum distress OR $A B$ post partum distress OR TI post-partum distress OR AB post-partum distress OR TI postnatal stress OR AB postnatal stress OR TI post natal stress OR AB post natal stress OR TI post-natal stress OR AB post-natal stress OR TI perinatal mental health OR AB perinatal mental health OR TI perinatal mental health problems OR AB perinatal mental health problems OR TI peri natal mental health problems OR AB peri natal mental health problems Or TI perinatal mental health problem OR AB perinatal mental health problem OR TI peri natal mental health problem OR AB peri natal mental health problem OR TI postnatal anxiety OR AB postnatal anxiety OR TI post natal anxiety OR AB post natal anxiety OR TI post-natal anxiety OR AB post-natal anxiety OR TI postpartum anxiety OR AB postpartum anxiety OR TI post partum anxiety OR AB post partum anxiety OR TI post-partum anxiety OR AB post-partum anxiety OR OR TI postnatal anxieties OR AB postnatal anxieties OR TI post natal anxieties OR AB post natal anxieties OR TI post-natal anxieties OR AB post-natal anxieties OR TI postpartum anxieties OR AB postpartum anxieties OR TI post partum anxieties OR AB post partum anxieties OR TI post-partum anxieties OR AB post-partum anxieties OR TI postnatal psychosis OR AB postnatal psychosis OR TI post natal psychosis OR AB post natal psychosis OR TI post-natal psychosis OR AB post-natal psychosis OR TI postpartum psychosis OR AB postpartum psychosis OR TI post partum psychosis OR $A B$ post partum psychosis OR TI post-partum psychosis OR AB post-partum psychosis OR

TI physical health OR AB physical health OR TI physical health problem OR AB physical health problem OR TI physical health problems OR AB physical health problems OR TI health problem OR AB health problem OR TI health problems OR AB health problems

\section{Results}

Results of search and selection strategy

Following removal of duplicates, a total of 2210 papers were retrieved (Fig. 1), and 978 were excluded on title screening. A further 1111 were excluded on abstract screening, and the remaining 121 papers were subjected to full text review. Citations not in English were screened at this stage using Google translate. A total of 12 papers appeared to meet the inclusion criteria for this review [25-36]; however, at data extraction stage it was realised that we were unable to abstract data from six papers [26, 29, 32-35] because the outcomes reported in these papers all related to management or treatments of $\mathrm{PPH}$, and no maternal physical or emotional health outcomes were reported. 
Table 2 Quality assessment tool - Modified Newcastle Ottawa Scale (NOS)

\begin{tabular}{|c|c|c|c|c|c|c|c|c|c|}
\hline \multirow{3}{*}{$\begin{array}{l}\text { Modified } \\
\text { Newcastle } \\
\text { Ottawa Scale } \\
\text { (NOS) }\end{array}$} & \multicolumn{9}{|c|}{ Quality Assessment Tool - Modified Newcastle Ottawa Scale (NOS) } \\
\hline & 1 & 2 & 3 & 4 & 5 & 6 & 7 & 8 & 9 \\
\hline & $\begin{array}{l}\text { Representativeness } \\
\text { of the exposed } \\
\text { cohort (postpartum } \\
\text { women) }\end{array}$ & $\begin{array}{l}\text { Do the } \\
\text { authors } \\
\text { provide a } \\
\text { definition } \\
\text { of PPH? }\end{array}$ & $\begin{array}{l}\text { Ascertainment } \\
\text { of exposure }\end{array}$ & $\begin{array}{l}\text { Assessment } \\
\text { of outcome }\end{array}$ & $\begin{array}{l}\text { Was the follow-up long } \\
\text { enough for outcome } \\
\text { to occur? (From birth } \\
\text { up to } 12 \text { months } \\
\text { postpartum or postnatal } \\
\text { period defined) }\end{array}$ & $\begin{array}{l}\text { Adequacy of } \\
\text { follow-up }\end{array}$ & $\begin{array}{l}\text { If necessary and/or } \\
\text { appropriate provide } \\
\text { details explaining } \\
\text { decision on adequacy } \\
\text { of follow-up }\end{array}$ & $\begin{array}{l}\text { How many stars } \\
\text { (a) does this } \\
\text { paper receive? }\end{array}$ & $\begin{array}{l}\text { Based on the overall } \\
\text { quality, do you wish } \\
\text { to include this paper? }\end{array}$ \\
\hline & $\begin{array}{l}\text { Truly } \\
\text { representative }\end{array}$ & $Y_{e s}^{a}$ & Secure record ${ }^{a}$ & $\begin{array}{l}\text { Independent } \\
\text { blind assessment }{ }^{\mathrm{a}}\end{array}$ & $Y_{e s}^{a}$ & $\begin{array}{l}\text { Complete } \\
\text { follow-up }\end{array}$ & & & $\checkmark=$ Yes, include \\
\hline & $\begin{array}{l}\text { Somewhat } \\
\text { representative }^{a}\end{array}$ & No & $\begin{array}{l}\text { Record linkage/ } \\
\text { Questionnaire }^{a}\end{array}$ & $\begin{array}{l}\text { Record linkage/ } \\
\text { Questionnaire }^{a}\end{array}$ & No & $\begin{array}{l}\text { Subjects lost } \\
\text { to follow-up }<20 \%{ }^{a}\end{array}$ & & & No $=$ Exclude \\
\hline & $\begin{array}{l}\text { Selected group } \\
\text { of users }\end{array}$ & & $\begin{array}{l}\text { Descriptive } \\
\text { written self-report }\end{array}$ & $\begin{array}{l}\text { Descriptive } \\
\text { self-report }\end{array}$ & & $\begin{array}{l}\text { Subjects lost to } \\
\text { follow-up }>20 \%\end{array}$ & & & $\begin{array}{l}\text { An overall rating of } 3 \\
\text { or less implies weak }\end{array}$ \\
\hline & No clear & & No description & No description & & No statement & & & $\begin{array}{l}\text { quality and should } \\
\text { be excluded }\end{array}$ \\
\hline & description & & & & & Not applicable ${ }^{a}$ & & & \\
\hline
\end{tabular}

${ }^{\mathrm{a}}$ One star awarded 


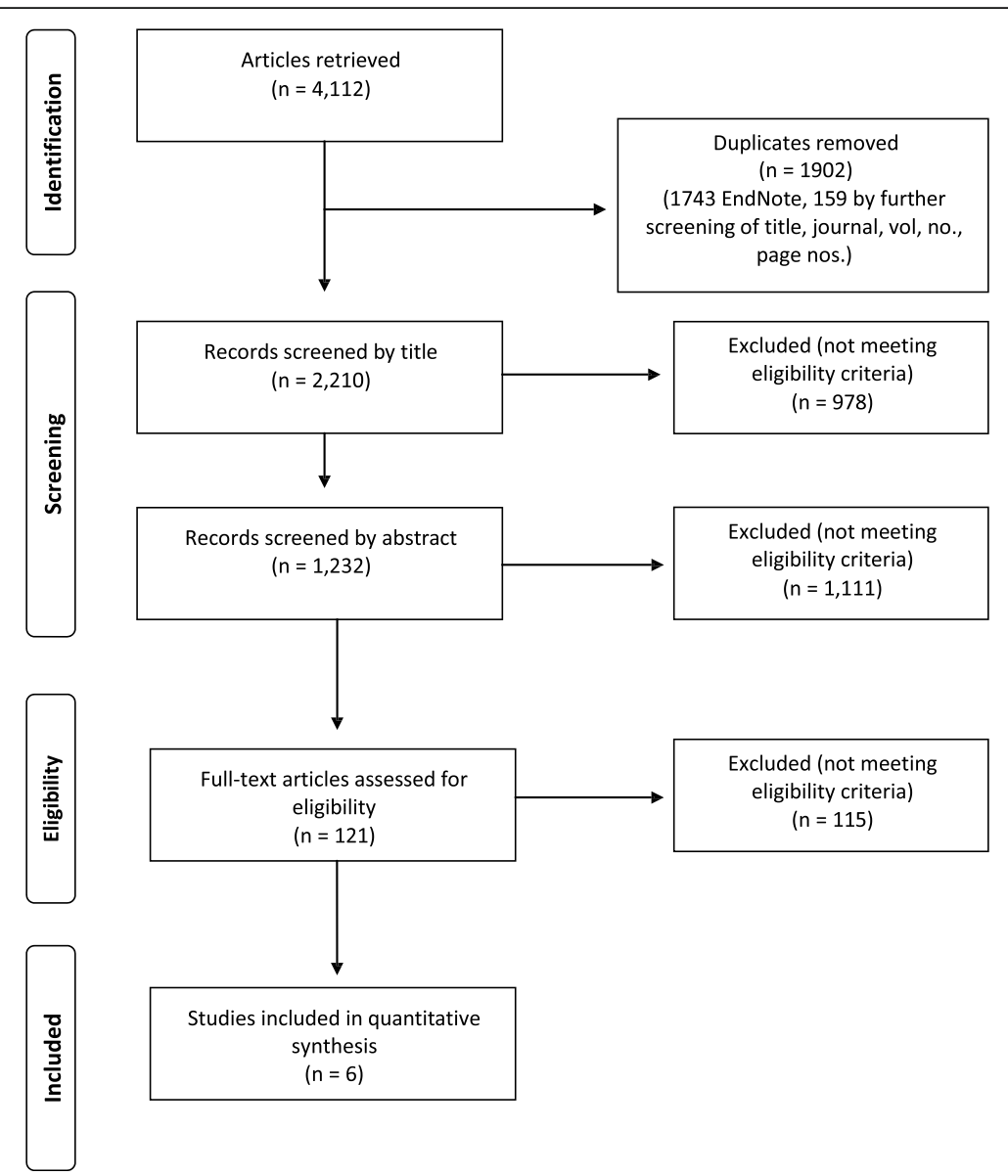

Fig. 1 Flow diagram of the search and selection process

\section{Characteristics of included studies}

The characteristics of the included studies are illustrated in Table 3. Five studies had the sequelae of $\mathrm{PPH}$ as their primary focus. The remaining study [27] focused on morbidity postnatally and we were able to extract data on the population of women who experienced a $\mathrm{PPH}$. The majority of studies took place between 1995 and 2007, with one study conducted in 1989 (Table 3). Four studies collected data over 1 to 3 years, and two studies over a 5-9 year time-frame (Table 3).

\section{Results of methodological quality assessment}

All included studies were judged as having strong or moderate methodological quality (Table 3). The two studies judged as having only moderate methodological quality were deemed so because $\mathrm{PPH}$ and/or the postnatal period were not defined in the publication.

\section{Results of data extraction}

The six included papers reported data from six individual studies conducted in seven countries. Five studies were conducted in OECD countries and one in a non-
OECD country. Detailed characteristics of included papers are presented in Table 3. Five studies reported the outcomes of primary PPH $[25,27,28,30,31]$ and one reported outcomes after secondary $\mathrm{PPH}$ [36].

\section{Definitions of PPH}

Studies varied in how they defined PPH, and these were compared with the definitions provided by WHO [1], RCOG [12], ICD-10 codes [13] and NICE [14]. One study, which was reporting outcomes after severe or major haemorrhage, stating it as $\geq 1,500 \mathrm{mls}$, defined $\mathrm{PPH}$ in the traditional manner, by amount of blood lost [27]. Two studies $[28,30]$ used the ICD-9 codes to define PPH which also includes "the amount that adversely affects the maternal physiology, such as blood pressure and haematocrit", although these parameters are not defined. Three studies defined PPH in a somewhat similar fashion, including treatment required, e.g., "bleeding ... persisting after manual exploration of the uterine cavity and requiring I.V. prostaglandin administration" [25], "findings of pelvic examination, condition of the uterus" [31], "a fall in 
Table 3 Characteristics and quality assessment of included studies

\begin{tabular}{|c|c|c|c|c|c|c|c|c|c|c|}
\hline Reference & Country & $\begin{array}{l}\text { OECD country } \\
\text { (at the time } \\
\text { research was } \\
\text { conducted) }\end{array}$ & $\begin{array}{l}\text { Year of } \\
\text { study }\end{array}$ & $\begin{array}{l}\text { Study } \\
\text { design }\end{array}$ & $\begin{array}{l}\text { ICD-10 (2010) or } \\
\text { similar definition } \\
\text { of PPH used }\end{array}$ & $\begin{array}{l}\text { Timing/severity/ } \\
\text { amount of PPH } \\
\text { used in definition }\end{array}$ & $\begin{array}{l}\text { Number and description } \\
\text { of participants included }\end{array}$ & $\begin{array}{l}\text { Data } \\
\text { collection }\end{array}$ & $\begin{array}{l}\text { Data reported and timing } \\
(\mathrm{HP},<1, \geq 1<3, \geq 3<6, \geq 6 \\
<12,=12 \text { months })\end{array}$ & $\begin{array}{l}\text { Quality } \\
\text { assessment }\end{array}$ \\
\hline $\begin{array}{l}\text { Chauleur et al. } \\
\text { (2008) [25] }\end{array}$ & France & Yes & 1999-2004 & $P, C$ & Similar & $\begin{array}{l}\text { Timing and severity } \\
\text { not amount of } \\
\text { bleeding }\end{array}$ & $\begin{array}{l}n=32,463 \text {, Primigravida } \\
\text { women (women in their } \\
\text { first intended pregnancy) }\end{array}$ & $\mathrm{D}, \mathrm{MR}$ & $\mathrm{HP}$ at $<1, \geq 1<3$ & Strong \\
\hline $\begin{array}{l}\text { Thompson et al. } \\
\text { (2011) [27] }\end{array}$ & $\begin{array}{l}\text { Australia } \\
\text { and New } \\
\text { Zealand }\end{array}$ & Yes & 2006-07 & $P, C$ & Similar & $\sqrt{ }$ & $\begin{array}{l}n=206 \text {, Primiparous and } \\
\text { Multiparous women who } \\
\text { returned the 1st survey }\end{array}$ & MR, Q & $\mathrm{HP}$ at $\geq 1<3, \geq 3<6$ & Strong \\
\hline $\begin{array}{l}\text { Bateman et al. } \\
\text { (2010) [28] }\end{array}$ & $\begin{array}{l}\text { United } \\
\text { States }\end{array}$ & Yes & 1995-2004 & $\mathrm{R}, \mathrm{C}$ & ICD-9 & $\sqrt{ }$ & $\begin{array}{l}n=25,654 \text {, Primiparous } \\
\text { and Multiparous women } \\
\text { who had a PPH }\end{array}$ & $\begin{array}{l}\text { MR (national } \\
\text { data) }\end{array}$ & $\mathrm{HP}$ at $<1$ & Moderate \\
\hline $\begin{array}{l}\text { Liu et al. } \\
\text { (2002) [30] }\end{array}$ & Canada & Yes & 1989-99 & $R, C$ & ICD-9 & $\sqrt{ }$ & $\begin{array}{l}n=2,652,726 \text {, Primiparous } \\
\text { and Multiparous women } \\
\text { who gave birth during a } \\
11 \text { year period }\end{array}$ & $\begin{array}{l}\text { MR (national } \\
\text { data) }\end{array}$ & $\mathrm{HP}$ at $\geq 1<3$ & Strong \\
\hline $\begin{array}{l}\text { Naz et al. } \\
\text { (2008) [31] }\end{array}$ & Pakistan & No & 2006 & $P, C$ & Not stated & $\begin{array}{l}\text { Severity of bleeding } \\
\text { but not amount or } \\
\text { timing }\end{array}$ & $\begin{array}{l}n=705 \text {, Primiparous and } \\
\text { Multiparous women who } \\
\text { had a Primary PPH }\end{array}$ & $\mathrm{MR}$ & $\mathrm{HP}$ at $<1$ & Moderate \\
\hline $\begin{array}{l}\text { Hoveyda and } \\
\text { MacKenzie (2001) [36] }\end{array}$ & England & Yes & 1996-98 & $\mathrm{R}$ & Similar & $\begin{array}{l}\text { Severity and timing } \\
\text { of bleeding but not } \\
\text { amount }\end{array}$ & $\begin{array}{l}n=19,136, \text { Primiparous } \\
\text { and Multiparous women } \\
\text { with Primary PPH, and those } \\
\text { with Secondary PPH }\end{array}$ & $\mathrm{MR}$ & $\mathrm{HP}$ at $<1$ & Strong \\
\hline
\end{tabular}


haemoglobin, for example, to $7 \mathrm{~g} / \mathrm{dl}$ and/or by $4 \mathrm{~g} / \mathrm{dl}$ or more" [25, 27].

\section{Outcomes of PPH}

One study reported on outcomes post secondary PPH, providing a definition similar to the RCOG's (2014) [36]. In total, 44 outcomes were reported in the six included papers (see Tables 4, 5 and 6 for a list and timeline of the health outcomes). The number of reported health problems varied from one (the rate of re-admission at $\geq 1$ but $<3$ months postpartum [30]), to 31 [27]. Four studies reported health outcomes in the 1st postpartum month $[25,28,31,36]$, two at $\geq 1<3[27,30]$, and one at $\geq 3<6$ [27]. Five reported outcomes following primary $\mathrm{PPH}[25,27,28,30,31]$ and one following both primary and secondary PPH [36].

The health outcomes reported in the early postpartum period tended to be acute, i.e., renal failure, respiratory failure, prolonged ventilation, coagulopathy/Disseminated Intravascular Coagulation (DIC), sepsis, anaemia, Superficial Venous Thrombosis and perforation of the uterus. Health problems reported in later months tended to be less severe, and some had become chronic.

Thompson et al. [27] was the only study that reported the prevalence of both emotional and physical health problems following a significant $(>1,500 \mathrm{mls})$ primary PPH between one and six months postpartum (Tables 5 and 6). The emotional health problems reported were anxiety, postnatal depression, fatigue, post-traumatic stress disorder and general health status, measured using Speilberger State-Trait Anxiety Inventory, Edinburgh Postnatal Depression Scale, Milligan's 10-item Scale for fatigue, 17-item PTSD Checklist and 360 item short form General Health Survey. The physical problems reported were infection (perineal, caesarean section (CS) wound, uterine, breast), pain (perineal, site of CS scar), urinary tract infection, incontinence (stress urinary, faeces, flatus), constipation, backache, headaches (frequent) and physical exhaustion. Women rated these as 'not a problem,' 'a minor problem, or 'a major problem'.

Only two studies compared women who had primary PPH with those who did not. Chauleur et al. [25] found that women who experienced $\mathrm{PPH}$ were at increased risk of superficial venous thrombosis (adjusted relative risk: 5.3, 95 \% CI 1.6 to 17). Hoveyda \& MacKenzie [36] analysed data from 657 women with primary PPH and found $33(5.02 \%)$ had a secondary PPH compared with only 99 out of 18,479 women who did not have primary $\mathrm{PPH}$ (0.54 \%), giving an odds ratio of 9.3 (95\% CI 6-2 to 14). One other study [27] provided data on those women re-admitted to hospital following a $\mathrm{PPH}$ at $\geq 1$ month and $<3$ months postpartum (4108 out of 113,861, $3.61 \%$ ). We compared these results with their data on the cohort of women who did not have PPH and were re-admitted (30,229 out of 2,538,865, $1.19 \%)$. The difference was statistically significant with an odds ratio of 3.1 (95 \% CI 3.01 to 3.21$), p<0.0001$.

Data on sub-groups of women, grouped by parity, age and BMI, following postpartum haemorrhage were not reported in any paper. Due to the different types of health outcomes reported in the individual studies, it was possible to pool results from only four studies, and only then by accepting the slightly differing definitions of PPH. Combining the results of Bateman et al. [28]

Table 4 Reported health problems up to one month postpartum

\begin{tabular}{|c|c|c|c|c|c|}
\hline References & $\begin{array}{l}\text { Chauleur et al. } \\
\text { (2008) [25] }\end{array}$ & $\begin{array}{l}\text { Bateman et al. } \\
\text { (2010) [28] }\end{array}$ & $\begin{array}{l}\text { Naz et al. } \\
\text { (2008) [31] }\end{array}$ & $\begin{array}{l}\text { Hoveyda \& MacKenzie } \\
\text { (2001) [36] }\end{array}$ & $\begin{array}{l}\text { Hoveyda \& MacKenzie } \\
\text { (2001) [36] }\end{array}$ \\
\hline Number of participants & $n=317$ & $n=25,654$ & $n=50$ & $n=657$ (primary PPH) & $n=132$ (secondary PPH) \\
\hline \multicolumn{6}{|c|}{ Reported Health Problems up to 1 month postpartum } \\
\hline Acute Renal Failure & & $82(0.32 \%)$ & $2(4 \%)$ & & \\
\hline Acute Respiratory Failure & & $105(0.41 \%)$ & & & \\
\hline Anaemia & & & $20(40 \%)$ & & \\
\hline Anaemia + DIC & & & $5(10 \%)$ & & \\
\hline Anaemia + DIC + ARF & & & $1(2 \%)$ & & \\
\hline Coagulopathy / DIC & & $445(1.73 \%)$ & $1(2 \%)$ & & \\
\hline Length of stay more than 7 days & & $656(2.56 \%)$ & & & \\
\hline Perforation of the uterus & & & & & $3(2.27 \%)$ \\
\hline Prolonged mechanical ventilation ( $>96 \mathrm{~h}$ ) & & $13(0.05 \%)$ & & & \\
\hline Secondary PPH & & & & $33(5.02 \%)$ & \\
\hline Sepsis / Infection & & $25(0.1 \%)$ & & & \\
\hline Shock + Anaemia & & & $2(4 \%)$ & & \\
\hline Superficial venous thrombosis & $3(0.9 \%)^{\mathrm{a}}$ & & & & \\
\hline
\end{tabular}


Table 5 Reported health problems $\geq 1$ month and $<3$ months postpartum

\begin{tabular}{lll}
\hline References & Thompson et al. & Liu et al. \\
& $(2011)[27]$ & (2002) [30] \\
Number of participants & $n=171$ & $n=113,861$ \\
\hline
\end{tabular}

Reported Health problems $\geq 1<3$ months postpartum

Anxiety: State Trait Anxiety Inventory 10 (median)

\begin{tabular}{|c|c|c|}
\hline $\begin{array}{l}\text { Depression: Edinburgh Postnatal } \\
\text { Depression Scale }>12\end{array}$ & $19(11 \%)$ & \\
\hline Post-traumatic stress disorder: PCL > 44 & $9(5 \%)$ & \\
\hline $\begin{array}{l}\text { Health Status: SF36 'somewhat worse } \\
\text { than } 1 \text { year ago' ago' }\end{array}$ & $46(26.9 \%)$ & \\
\hline $\begin{array}{l}\text { Health Status: SF36 'much worse than } \\
1 \text { year ago' }\end{array}$ & $14(8.2 \%)$ & \\
\hline \multicolumn{3}{|l|}{ hysical problems: } \\
\hline i. Painful perineum (vaginal births only) & $54 / 103(52.5 \%)$ & \\
\hline ii. Perineal infection (vaginal births only) & 6/104 (5.8 \%) & \\
\hline iii. Pain at CS site (CS births only) & $53 / 65(81.5 \%)$ & \\
\hline iv. CS wound infection (CS births only) & $12 / 65(18.5 \%)$ & \\
\hline v. Uterine infection & $10(5.9 \%)$ & \\
\hline vi. Urinary tract infection & $11(6.5 \%)$ & \\
\hline vii. Stress urinary incontinence & $57(33.5 \%)$ & \\
\hline viii. Constipation & $81(47.6 \%)$ & \\
\hline ix. Incontinence of faeces & $10(5.9 \%)$ & \\
\hline$x$. Incontinence of flatus & $31(18.5 \%)$ & \\
\hline xi. Breast infection/ mastitis & $37(21.8 \%)$ & \\
\hline xii. Backache & $92(54.7 \%)$ & \\
\hline xiii. Headache (frequent) & 49 (29.2\%) & \\
\hline xiv. Physical exhaustion & $119(71.2 \%)$ & \\
\hline $\begin{array}{l}\text { Re-admission } \\
\text { Reasons: }\end{array}$ & $17(10 \%)$ & $4108(3.6 \%)$ \\
\hline i. Mastitis/Breast abscess & $4 / 17$ & \\
\hline $\begin{array}{l}\text { ii. Bleeding/Retained of products } \\
\text { of conception }\end{array}$ & $3 / 17$ & \\
\hline iii. Low haemoglobin/transfusion & $3 / 17$ & \\
\hline iv. Problems with perineal stitches & $2 / 17$ & \\
\hline $\begin{array}{l}\text { v. Postnatal depression, } \\
\text { hyperventilation, pleuritic pain, } \\
\text { blood clot on liver, severe migraine, } \\
\text { neonatal jaundice }\end{array}$ & 1/17 (each) & \\
\hline
\end{tabular}

CS caesarean section

and Naz et al. [31] gave a total of 25,704 women. Acute renal failure occurred in 84 women $(0.33 \%)$ and coagulopathy in $446(1.74 \%)$. However, it should be noted that the study by Naz et al. [31] was conducted on 50 women who had primary PPH, of whom 10 (20 \%) had a subtotal hysterectomy performed, a population rate of peripartum hysterectomy $(1.42 \%)$ far in excess of the prevalence found in other countries. It is likely that the high incidence of acute renal failure and other
Table 6 Reported health problems $\geq 3$ month and $<6$ months postpartum

\begin{tabular}{ll}
\hline References & $\begin{array}{l}\text { Thompson et al. } \\
(2011)[27]\end{array}$ \\
Number of participants & $n=171$ \\
\hline Anxiety: State Trait Anxiety Inventory (median) & 10 \\
Depression: Edinburgh Postnatal Depression Scale > 12 & $21(13 \%)$ \\
Post-traumatic stress disorder: PCL > 44 & $5(3 \%)$ \\
Health Status: SF36 'somewhat worse than 1 year ago' & $51(30.7 \%)$ \\
Health Status: SF36 'much worse than 1 year ago' & $10(6 \%)$ \\
Physical problems: & \\
i. Painful perineum (vaginal births only) & $25 / 100(25 \%)$ \\
ii. Perineal infection (vaginal births only) & 0 \\
iii. Pain at CS site (CS births only) & $29 / 60(48.3 \%)$ \\
iv. CS wound infection (CS births only) & $5 / 61(8.2 \%)$ \\
v. Uterine infection & $4(2.4 \%)$ \\
vi. Urinary tract infection & $6(3.7 \%)$ \\
vii. Stress urinary incontinence & $42(25.6 \%)$ \\
viii. Constipation & $39(24 \%)$ \\
ix. Incontinence of faeces & $5(3 \%)$ \\
x. Incontinence of flatus & $26(16.1 \%)$ \\
xi. Breast infection/ mastitis & $22(13.6 \%)$ \\
xii. Backache & $99(60 \%)$ \\
xiii. Headache (frequent) & $51(30.9 \%)$ \\
xiv. Physical exhaustion & $87(52.7 \%)$ \\
Re-admission & $4(2 \%)$ \\
Reasons: & $2 / 4$ \\
i. Bleeding/ Retained of products of conception \\
ii. Post-natal depression & $1 / 4$ \\
iii. Cholecystitis & $1 / 4$ \\
\hline
\end{tabular}

CS caesarean section

complications seen in this study are due to the surgical procedure, rather than $\mathrm{PPH}$ per se. The benefit of combining these results is that a more realistic overall prevalence is reached.

The combined results of Liu et al. [30] and Thompson et al. [27] in relation to those re-admitted to hospital following a PPH at $\geq 1$ month and $<3$ months postpartum, gave a total of 4125 re-admissions out of 114,032 (3.6\%).

\section{Discussion}

There are few papers that report on health outcomes following a postpartum haemorrhage, despite the reported increase in the incidence of PPH and more severe PPH, across the globe [8-10]. Many papers focus only on the risks for, and prevention of, maternal mortality due to $\mathrm{PPH}$, with scant regard for surviving women's physical 
and emotional state. Women's health and level of possible morbidity post PPH is thus virtually unknown.

From the 2210 papers identified in this review, only six reported on outcomes following PPH. Of these, we were unable to pool and meta-analyse most data because of differing definitions of $\mathrm{PPH}$, differing timing of measurement or differing outcomes measured. Those that could be pooled demonstrated a $0.33 \%$ rate of acute renal failure and a $1.74 \%$ rate of coagulopathy. The readmission rate to hospital following a $\mathrm{PPH}$ between 1 and 3 months postpartum was $3.6 \%$, an appreciable indication of underlying physical problems. Single studies included in this review showed other types of morbidity to be common; for example, depression (13\%) and a health status at 6 months postpartum, as measured by SF36, as 'much worse than 1 year ago' (6 \%) [27].

Few studies of emotional sequelae of PPH within the six month postpartum period were found. Some work has shown that $68 \%$ of 68 women surviving severe PPH had negative memories of the event (such as fear of dying (35\%)) when interviewed 4 to 17 years later) [5]. Sixty percent of the 15 women who went on to have another pregnancy suffered severe anxiety throughout, indicating the tenacity of such feelings. If emotional morbidity from PPH could be detected in the early postpartum months, and treated, it is possible that such long-term psychological disability could be averted. The first step, however, is to measure the prevalence and severity of such morbidity and an observational study is in progress in France that may provide more information in this area [37].

Secondary PPH is poorly defined, yet it seems illogical to define it as a blood loss of $500 \mathrm{mls}$, the amount used to define primary $\mathrm{PPH}$, because this is considered to be in excess of what is considered a physiologically normal blood loss during the third stage of labour. We suggest that the definition should more correctly be: "any blood loss from the gentital tract in excess of normal lochia at any time period after $24 \mathrm{~h}$ post birth to 6 weeks postpartum."

It is clear that more research is needed, to ascertain the amount and degree of both physical and emotional postpartum morbidity following PPH. Before that, though, it is important to develop a core outcomes set [38] for measuring maternal health outcomes postpartum, perhaps as a subset of the maternity core outcome set developed by Devane et al. [39].

\section{Strengths and limitations of this review}

The findings from this review are based on a comprehensive systematic literature search of seven electronic databases, and rigorous critical appraisal of included studies. Two studies with large sample sizes $[28,30]$ influenced the overall results. Unfortunately there was high heterogeneity between all studies, which is a major limitation. We tried to be as inclusive as possible, but we were unable to pool and meta-analyse most of the findings, thus limiting the review's potential to add new knowledge to clinical practice. Despite this limitation, the findings demonstrate that the prevalence of physical and emotional health problems experienced by women post PPH remain largely unknown, indicating the need for further research in this area.

\section{Conclusion}

This review demonstrates that there are few studies examining the prevalence of maternal physical and health problems post $\mathrm{PPH}$, and those that were found tended to be either small and/or focussed on a limited number of morbidities. PPH, estimated to affect as many as $8-11$ million women annually, is likely to impact negatively on women's health, and it is conceivable that existing health problems experienced may be exacerbated. As these health problems remain virtually unknown, however, many women may be suffering sequelae from PPH during the postpartum period in the community, without proper diagnosis or treatment. Further research is required, but because of the variations in definitions used, and outcomes reported, studies will require a clear definition of $\mathrm{PPH}$, and valid and reliable measuring instruments, based on a core outcome set, to ascertain the timing, prevalence and severity of health problems.

\section{Additional file}

Additional file 1: Search strings for databases. (DOCX 24.7 kb)

\section{Acknowledgements}

We are grateful to the Health Research Board of Ireland for funding this review. The opinions expressed in this review are those of the authors and are not necessarily those of the funder.

Availability of data and materials

All data used are presented and included within the manuscript (and its Additional file 1).

\section{Authors' contributions}

$M C$ and DD developed the search strategy, which was reviewed by CB. MC conducted the search. All authors independently selected the papers for inclusion. MC and DD independently assessed the quality of the included papers. MC and DD independently extracted the data and CB checked these data for accuracy. MC drafted the paper and all authors commented and agreed the paper for submission.

\section{Competing interests}

The authors declare that they have no competing interests.

Consent for publication

Not applicable.

Ethics approval and consent to participate Not applicable. 


\section{Author details}

'School of Nursing and Midwifery, Trinity College Dublin, 24 D'Olier Street, Dublin DO2 T283, Ireland. '2 Institute of Health and Care Sciences, Sahlgrenska Academy, University of Gothenburg, Gothenburg, Sweden.

\section{Received: 24 April 2016 Accepted: 22 August 2016}

Published online: 05 September 2016

\section{References}

1. World Health Organisation. WHO recommendations for the prevention and treatment of postpartum haemorrhage. 2012. http://apps.who.int/iris/ bitstream/10665/75411/1/9789241548502_eng.pdf. Accessed 30 Aug 2016.

2. Knight M, Tuffnell D, Kenyon S, Shakespeare J, Gray R, Kurinczuk JJ, on behalf of MBRRACE-UK. Saving lives, improving mothers' care - surveillance of maternal deaths in the UK 2011-13 and lessons learned to inform maternity care from the UK and Ireland confidential enquiries into maternal deaths and morbidity 2009-13. Oxford: National Perinatal Epidemiology Unit, University of Oxford; 2015.

3. Rocha Filho EA, Costa ML, Cecatti JG, Parpinelli MA, Haddad SM, Pacagnella RC, Sousa MH, Melo Jr EF, Surita FG, Souza JP. Severe maternal morbidity and near miss due to postpartum haemorrhage in a national multicenter surveillance study. Int J Gynecol Obstet. 2015;128(2):131-6.

4. Wagner KS, Ronsmans C, Thomas SL, Calvert C, Adler A, Ganaba R, Goufodji S, Filippi V. Women who experience obstetric haemorrhage are at higher risk of anaemia, in both rich and poor countries. Trop Med Int Health. 2012; 17(1):9-22.

5. Sentilhes L, Gromez A, Clavier E, Resch B, Descamps P, Marpeau L. Longterm psychological impact of severe postpartum haemorrhage. Acta Obstet Gynecol Scand. 2011;90(6):615-20.

6. Furuta M, Sandall J, Bick D. Women's perceptions and experiences of severe maternal morbidity - A synthesis of qualitative studies using a metaethnographic approach. Midwifery. 2014;30(2):158-69.

7. Likis FE, Sathe NA, Morgans AK, Hartmann KE, Young JL, Carlson-Bremer D, Schorn M, Surawicz T, Andrews J. Management of postpartum hemorrhage. Comparative Effectiveness Review No. 151. (Prepared by the Vanderbilt Evidence-based Practice Center under Contract No. 290-2012-00009-I.) AHRQ Publication No. 15-EHC013-EF. Rockville: Agency for Healthcare Research and Quality; 2015. http://www.effectivehealthcare.ahrq.gov/ehc/products/552/2078/ hemorrhage-postpartum-report-150427.pdf.

8. Paterson-Brown S, Bamber J, on behalf of the MBRRACE-UK haemorrhage chapter writing group. Prevention and treatment of haemorrhage. In: Knight M, Kenyon S, Brocklehurst P, Neilson J, Shakespeare J, Kurinczuk JJ, on behalf of MBRRACE-UK, editors. Saving lives, improving mothers' care lessons learned to inform future maternity care from the UK and Ireland Confidential Enquiries into maternal deaths and morbidity 2009-12. Oxford: National Perinatal Epidemiology Unit, University of Oxford; 2014. p. 45-55.

9. Lutomski J, Byrne B, Devane D, Greene R. Increasing trends in atonic postpartum haemorrhage in Ireland: an 11-year population based cohort study. BJOG. 2012;119:306-14. doi:10.1111/j.1471-0528.2011.03198.x.

10. Ford JB, Patterson JA, Seeho SK, Roberts CL. Trends and outcomes of postpartum haemorrhage, 2003-2011. BMC Pregnancy Childbirth. 2015;15:334.

11. Sheehan S. Coombe Women and Infants University Hospital annual clinical report 2014. Dublin: Coombe Women and Infants University Hospital; 2014. http://www.coombe.ie/index.php?nodeld=110. Accessed 5 Mar 2016.

12. Royal College of Obstetricians and Gynaecologists (RCOG). Prevention and management of postpartum haemorrhage Green-top Guideline 52. 2009. https://www.rcog.org.uk/globalassets/documents/guidelines/ gt52postpartumhaemorrhage0411.pdf. Accessed 5 Mar 2016.

13. ICD-10. ICD-10-CM diagnosis code. 2016. http://www.icd10data.com/ ICD10CM/Codes/O00-O9A/O60-077/O72. Accessed 5 Mar 2016.

14. National Institute for Health and Care Excellence (NICE). Intrapartum care for health for healthy women and babies. 2014. https://www.nice.org.uk/ guidance/cg190. Accessed 5 Mar 2016.

15. Corcoran P, Manning E, Meaney S, Greene RA, on behalf of the Maternal Morbidity Advisory Group. Severe maternal morbidity in ireland annual report 2012 and 2013. Cork: National Perinatal Epidemiology Centre; 2015.

16. Scottish Confidential Audit of Severe Maternal Morbidity (SCASMM). Scottish confidential audit of severe maternal morbidity: reducing avoidable harm 10th annual report. 2014. http://www.healthcareimprovementscotland.org/ our_work/reproductive,_maternal_child/programme_resources/scasmm. aspx. Accessed 5 Mar 2016.
17. Diaz V, Abalos E, Carroli G. Methods for blood loss estimation after vaginal birth. Cochrane Database Syst Rev. 2014;2:CD010980. doi:10.1002/14651858. CD010980.

18. Pacagnella RC, Souza JP, Durocher J, Perel P, Blum J, et al. A systematic review of the relationship between blood loss and clinical signs. PLoS One. 2013:8:3. doi:10.1371/journal.pone.0057594.

19. Schorn MN. Measurement of blood loss: review of the literature. J Midwifery Womens Health. 2010;55(1):20-7.

20. Stafford I, Dildy GA, Clark SL, et al. Visually estimated and calculated blood loss in vaginal and cesarean delivery. Am J Obstet Gynecol. 2008; 199:519.e1-7.

21. Vanderkruik RC, Tunçalp Ö, Chou D, Say L. Framing maternal morbidity: WHO scoping exercise. BMC Pregnancy Childbirth. 2013;13(1):1-15. doi:10.1186/1471-2393-13-213.

22. Horsley T, Dingwal O, Sampson M. Checking reference lists to find additional studies for systematic reviews. Cochrane Database Syst Rev. 2011; 8:MR000026. doi:10.1002/14651858.MR000026.pub2.

23. Higgins JPT, Green S, (editors). Cochrane handbook for systematic reviews of interventions Version 5.1.0 [updated March 2011]. The Cochrane Collaboration. 2001. http://handbook.cochrane.org/. Accessed 5 Mar 2016.

24. Wells G, Shea B, O'Connell D, Welch JV, Losos M, Tugwell P. The NewcastleOttawa Scale (NOS) for assessing the quality of nonrandomised studies in meta-analyses. 2008. http://www.ohri.ca/programs/clinical_epidemiology/ oxford.asp. Accessed 5 Mar 2016.

25. Chauleur C, Cochery-Nouvellon E, Mercier E, Aya G, Mares P, Mismetti P, Lissalde-Lavigne G. Gris first cohort. Thromb Haemost. 2008;100(5):773-9.

26. Jacobson L, Kaij L, Nilsson A. Course and outcome of post-partum period from a gynaecological and general somatic standpoint. Acta Obstet Gynecol Scand. 1967;46(2):183-203.

27. Thompson JF, Roberts CL, Ellwood DA. Emotional and physical health outcomes after significant primary post-partum haemorrhage (PPH): a multicentre cohort study Aust NZ. J Obstet Gynaecol. 2011;51(4):365-71.

28. Bateman BT, Berman MF, Riley LE, Leffert LR. The epidemiology of postpartum hemorrhage in a large, nationwide sample of deliveries. Anesth Analg. 2010;110(5):1368-73.

29. Soltan Mohamed H, Sadek RR. Experience managing postpartum hemorrhage at Minia University Maternity Hospital, Egypt: No mortality using external aortic compression. J Obstet Gynaecol Res. 2011;37(11):1557-63.

30. Liu SL, Heaman M, Kramer MS, Demissie K, Wen SW, Marcoux S. Length of hospital stay, obstetric conditions at childbirth, and maternal readmission: a population-based cohort study. Am J Obstet Gynecol. 2002;187(3):681-7.

31. Naz H, Sarwar I, Fawad A, Nisa AU. Maternal morbidity and mortality due to primary PPH-experience at Ayub Teaching Hospital Abbottabad. J Ayub Med Coll Abbottabad. 2008;20(2):59-65.

32. Ade-Conde JA, Alabi O, Higgins S, Visvalingam G. Maternal post natal hospital readmission-trends and association with mode of delivery. Ir Med J. 2011;104(1):17-20

33. Fullerton G, Danielian PJ, Bhattacharya S. Outcomes of pregnancy following postpartum haemorrhage. BJOG. 2013;120(5):621-7.

34. Marchant S, Alexander J, Garcia J. Postnatal vaginal bleeding problems and general practice. Midwifery. 2002;18(1):21-4.

35. Furuta M, Sandall J, Cooper D, Bick D. The relationship between severe maternal morbidity and psychological health symptoms at 6-8 weeks postpartum: a prospective cohort study in one English maternity unit. BMC Pregnancy Childbirth. 2014;14:133. doi:10.1186/1471-2393-14-133.

36. Hoveyda F, MacKenzie IZ. Secondary postpartum haemorrhage: incidence, morbidity and current management. BJOG. 2001;108(9):927-30.

37. Gayat E, Mebazaa A. Emotional and cardiac impact of postpartum haemorrhage (HELP-MOM). CLinicalTrials.gov. 2014. https://clinicaltrials.gov/ ct2/show/NCT02118038 Accessed 21 Feb 2016.

38. Williamson P, Clarke M. The COMET (Core Outcome Measures in Effectiveness Trials) Initiative: its role in improving Cochrane Reviews [editorial]. Cochrane Database Syst Rev. 2012;(4): 10.1002/14651858.ED000041.

39. Devane D, Begley CM, Clarke M, Horey D, OBoyle C. Evaluating maternity care: a core set of outcome measures. Birth. 2007;34(2):164-71. 